\title{
Design of acceleration/deceleration profiles in motion control based on digital FIR filters
}

\author{
Chin-Sheng Chen, An-Chen Lee* \\ Department of Mechanical Engineering, National Chiao Tung University, 1001 Ta Hsueh Road, Hsinchu 300-10, \\ Taiwan, R.O.C.
}

Received 14 April 1997; final form 28 July 1997

\begin{abstract}
In motion control, the reference commands should pass through the acceleration/deceleration (Acc/Dec) processor before being fed into the servo loop. Consequently, not only can a smooth motion profile be obtained but undesirable vibration can be avoided. In this study, we develop a systematic design procedure for the Acc/Dec processor based on the discrete time finite-impulse-response (FIR) filter design technique with some necessary conditions. Compared with the conventional Acc/Dec scheme, the FIR Acc/Dec scheme poses the advantage that the contouring error can be analyzed. Also, the contouring error caused by the conventional Acc/Dec scheme is evaluated by demonstrating a mapping technique from the conventional Acc/Dec scheme to the FIR Acc/Dec scheme. The proposed Acc/Dec algorithm is quite flexible and efficient, creating space for users to use more sophisticated profiles to more efficiently match the system constraints with minimum contouring errors. Simulation and experimental results verify that the proposed Acc/Dec processor can effectively improve the motion accuracy. (C) 1998 Elsevier Science Ltd. All rights reserved.
\end{abstract}

\section{Introduction}

Demands for high accuracy and high speed motion in a motion mechanism are increasingly stringent. To satisfy these demands, many investigators have developed and refined those tech-

\footnotetext{
* Corresponding author.
} 
niques, including those for mechanical structure, the efficient servo control algorithms, and the interpolation algorithms.

As for interpolation algorithms, two techniques, i.e., the reference pulse [1,2] and the sample data [1,3] schemes, are generally employed. The motion profiles generated using the above two schemes have been studied extensively. Those schemes focus primarily on how to accurately depict the motion profile without considering the corresponding system limitations. Therefore, as change of interpolation output between two sampling intervals becomes larger, an enormous step change in the servo loop occurs. This enormous step change of the servo loop not only incurs the contouring error, but also excites the undesirable modes, which subsequently cause the shock and vibration of the mechanism. Furthermore, damaging may occur. Hence, the reference commands should pass through the acceleration/deceleration (Acc/Dec) processor before fed into the servo loop. Consequently, not only can a smooth motion profile be obtained but undesirable vibration can be avoided.

Despite recognition of its importance, Acc/Dec processor has received little attention in previous literature. In the conventional Acc/Dec design, with given constraints for the initial and final positions, primary emphasis is placed on selecting a class of polynomial functions of degree $\mathrm{n}$ or less such that the required positions, velocities, and accelerations at these points are satisfied. The more constraints to be specified implies the higher order of a polynomial to meet them [46]. However, a serious limitation of conventional Acc/Dec is that the contouring errors cannot be analyzed. In addition to this limitation, employing this method to develop sophisticated acceleration profiles that closely match system limits for high performance motion control is extremely difficult.

This study develops a systematic design procedure for the Acc/Dec processor based on the discrete time finite-impulse-response (FIR) filter design technique with some necessary conditions. Furthermore, the contouring error caused by the FIR Acc/Dec scheme is analyzed. Also, the contouring error caused by the conventional Acc/Dec scheme is evaluated by demonstrating a mapping technique from the conventional Acc/Dec scheme to the FIR Acc/Dec scheme. The proposed Acc/Dec algorithm is quite flexible and efficient, creating space for users to use more sophisticated profiles to more efficiently match the system constraints with minimum contouring errors. Simulation and experiments results verify that the proposed Acc/Dec processor can effectively improve the motion accuracy.

\section{Acc/Dec design based on a digital FIR filter}

As shown in Fig. 1, according to the geometrical relationship, the velocity of each axis for a multi-axis motion system is generated from the interpolator. The velocity of each axis is then individually fed into the Acc/Dec processor. Using this scheme, we can always obtain a smooth velocity command for each axis.

In the stream of command generation, the Acc/Dec processor can be regarded as a post-processor for the interpolator. The output commands of Acc/Dec processor, which are capable of producing a smooth motion profile when the velocity set point is overrided. Consequently, not only can the motion accuracy be improved, but shock and vibration of the mechanism can be avoided.

An Acc/Dec algorithm is usually realized digitally in NC machines in practical implementation. 


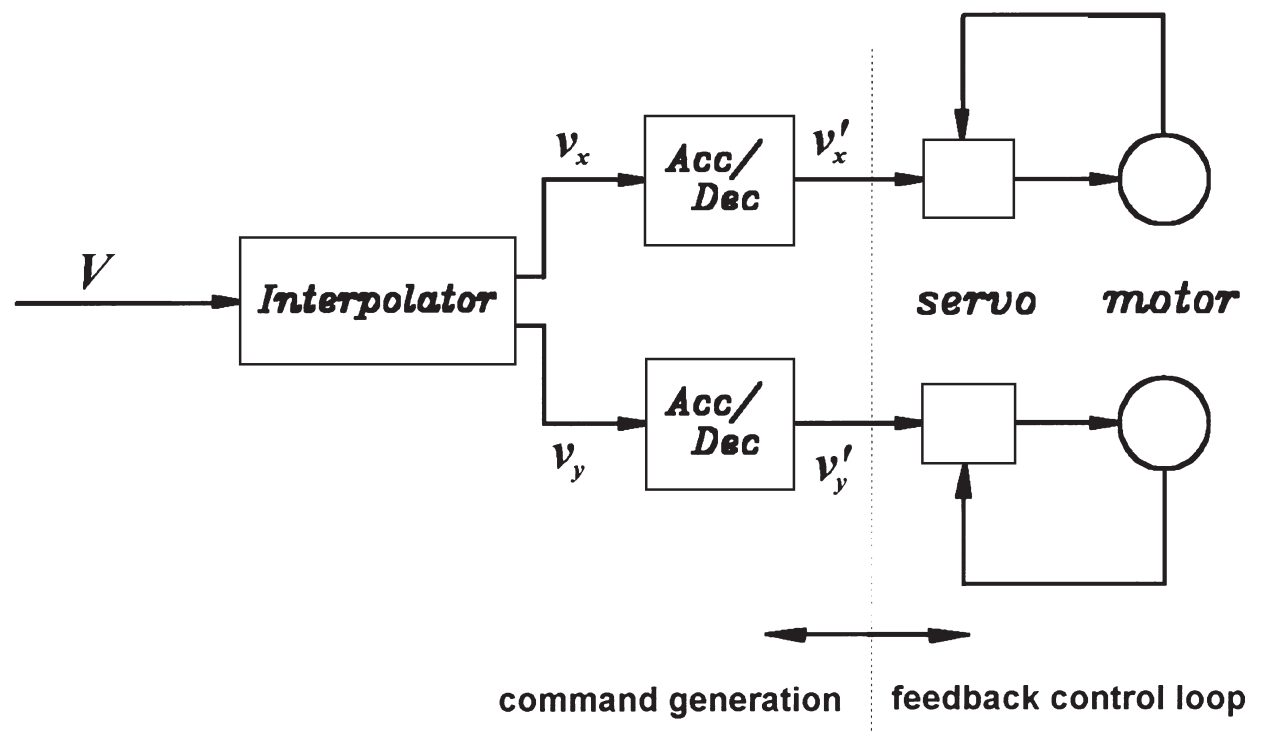

Fig. 1. Layouts of the Acc/Dec processor and the interpolator.

Here, $V[k T]$ is assumed here to be the velocity command before smoothing and $V^{\prime}[k T]$ is the current velocity command from the Acc/Dec processor. By applying the concept of moving average, the design for various Acc/Dec velocity curves becomes feasible as stated in the following.

Let $V^{\prime}[k T]$ be manipulated as a weighted moving average of the recent $\mathrm{n}$ velocity commands $V[k T], V[(k-1) T], V[(k-2) T], \ldots \ldots . V[(k-n-1) T]$, associated with the appropriate weighted coefficients $b_{i}\left(b_{0}, b_{1}, \ldots \ldots b_{n-1}\right)$. Consequently, the input-output velocity relationship for the Acc/Dec processor can be expressed as

$$
V^{\prime}[k T]=\frac{1}{b_{s}} \sum_{i=0}^{n-1} b_{i} \cdot V[(k-i) T]
$$

or

$$
V^{\prime}[k]=\frac{1}{b_{s}} \sum_{i=0}^{n-1} b_{i} \cdot V[k-i]
$$

where $V^{\prime}[k]$ and $V[k]$ denote the velocity of each axis and $b_{s}$ is the position convergent factor which will be determined later. With the help of $\mathrm{z}$ transformation, the behavior of the Acc/Dec processor as described in Eq. (1a) is then equivalent to a dynamic system of order $n-1$.

$$
D(z) \equiv \frac{V^{\prime}(z)}{V(z)}=\frac{1}{b_{s}} \sum_{i=0}^{n-1} b_{i} \cdot z^{-i}
$$

where $\mathrm{z}$ is a unit delay operator, and $V^{\prime}[\mathrm{z}]$ and $V[\mathrm{z}]$ are the $\mathrm{z}$ transformations of $V^{\prime}[k]$ and $V[k]$, respectively. Figure 2 presents the proposed Acc/Dec scheme. 


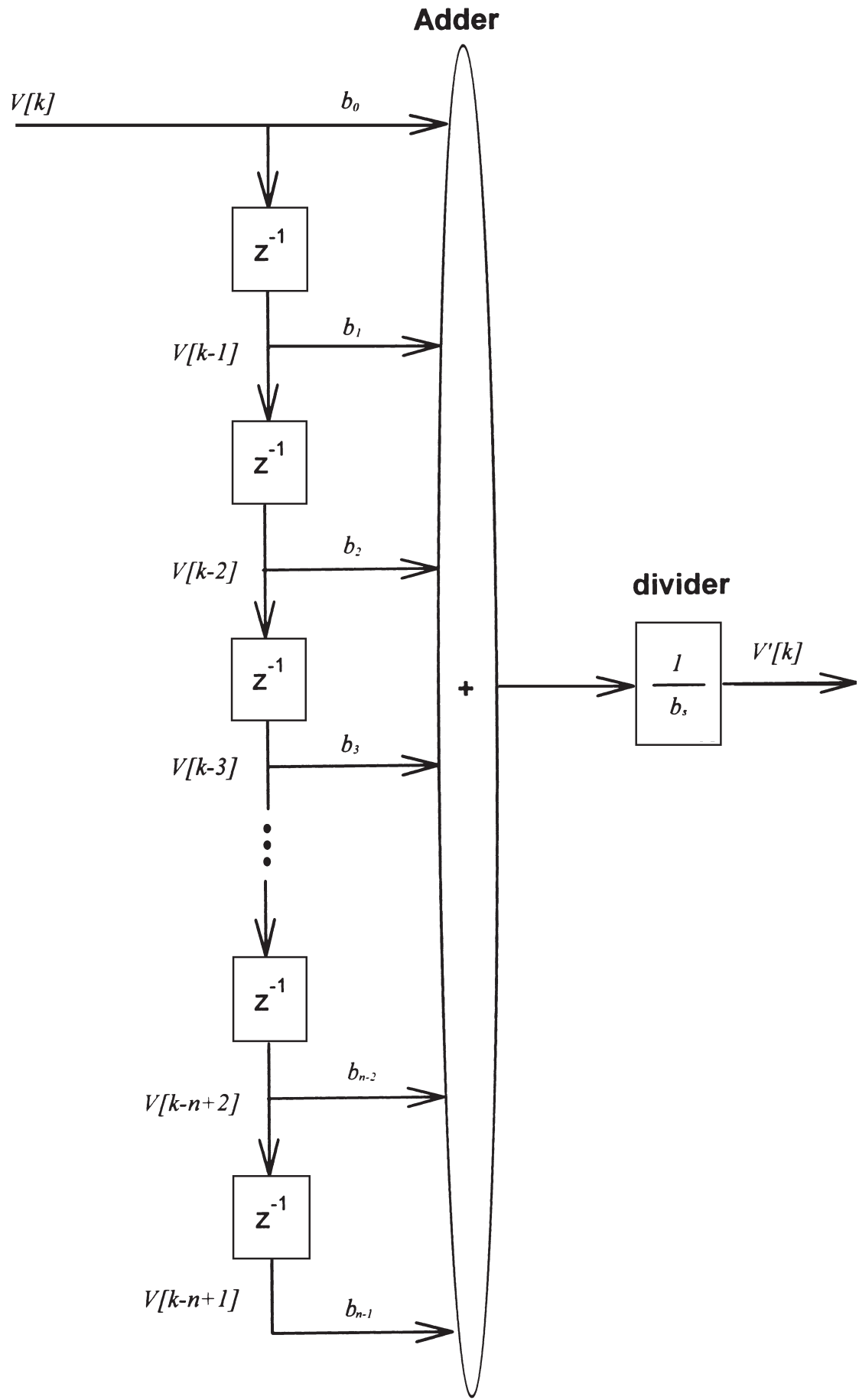

Fig. 2. The proposed Acc/Dec scheme. 
In the Acc/Dec design, three conditions must be considered. First, the final position after Acc/Dec processor must converge to the desired position in motion control. For the illustrative purposes, as shown in Fig. 3, two velocity profiles represent input and output curves of the Acc/Dec processor. Assume that the velocity command into the Acc/Dec sustains a period of mT; then, the velocity output out of the Acc/Dec sustains a period of $(m+n) T$. To have equal excursion in both input and output, the following condition must be satisfied:

$$
\sum_{k=0}^{m} V[k] \cdot T=\sum_{k=0}^{m+n} V^{\prime}[k] \cdot T
$$

By the property of $\mathrm{z}$ transform, Eq. (3) implies that

$$
\lim _{z \rightarrow 1} V(z)=\lim _{z \rightarrow 1} V^{\prime}(z)
$$

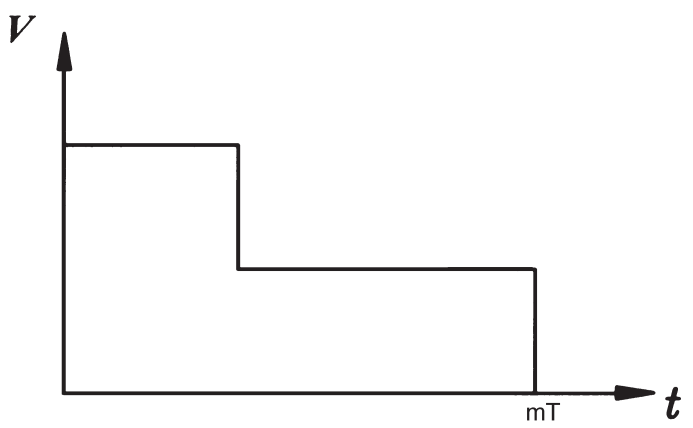

(a)

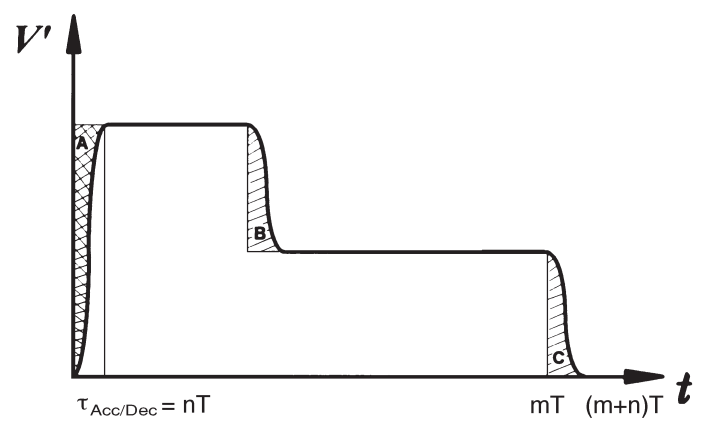

(b)

Fig. 3. Input and output velocity profiles of the Acc/Dec processor. (a) Velocity profile into the Acc/Dec. (b) Velocity profile out of the Acc/Dec. 
Substituting Eq. (4) into Eq. (2) yields a relationship between $b_{s}$ and the weighted coefficient $b_{i}$, or

$$
b_{s}=\sum_{i=0}^{n-1} b_{i}
$$

The above condition guarantees the final position after Acc/Dec converge to the desired position. Restated, this condition will cause the cross-hatched area in Fig. $3 \mathrm{~b}$ to be equal to two separate hatched areas (i.e., $\mathrm{A}=\mathrm{B}+\mathrm{C}$ ). For the convenience in later discussion, Acc/Dec time constant $\tau_{\text {Acc/Dec }}$ is defined here as

$$
\tau_{\mathrm{Acc} / \mathrm{Dec}}=n \cdot T
$$

where $\mathrm{T}$ is the sampling time. As shown in Fig. 3b, the time constant is fixed in the overall tracking path, which is proportional to the number of buffers in implementation.

Second, all the weighted coefficients $b_{i}$ s should be semi-positive (i.e., $b_{i} \geq 0$ ); otherwise, the velocity curve after the Acc/Dec will appear in an oscillatory pattern when the velocity is overrided. Fig. 4 illustrates this phenomenon, where Fig. 4 a shows the impulse response of D(z), i.e., the coefficients $b_{i}$ of the dynamic system $\mathrm{D}(\mathrm{z})$. Also, Fig. $4 \mathrm{~b}$ shows the normalized unit velocity command and response. Those figures clearly indicate that the velocity profile out of the Acc/Dec will have an oscillatory nature if some weighted coefficients $b_{i}$ s are negative. This oscillation in velocity profile is not desirable, because it will decrease the motion accuracy.

The third condition derives from the practical limitation in designing an Acc/Dec curve arising from the system limitations. From Eq. (1b) and Fig. 6, the acceleration of each axis can be derived as

$$
a^{\prime}[k]=V[k] \cdot \frac{b_{k-1}}{T \cdot b_{s}} \quad 1 \leq k \leq n
$$

Consider the system limitations: The velocity $V^{\prime}[k]$ must be less than the velocity limitation $V_{\text {Max }}$, the force $F[k]=m a^{\prime}[k]+c V^{\prime}[k]$ less than the force limitation $F_{\text {Max }}$, and the power $P[k]=$ $F[k] \cdot V^{\prime}[k]$ also less than the power limitation $P_{\text {Max }}$, where $\mathrm{m}$ is the equivalent mass of load and $\mathrm{c}$ is the equivalent viscous damping of the controlled plant.

Various Acc/Dec velocity profiles can be designed by tuning the weighted coefficient $b_{i}$ s. For example, there are two cases presented in Fig. 5 where the velocity, acceleration and weighted coefficient $b_{i}$ for two Acc/Dec processors are provided. Fig. 5b shows the velocity from a linear Acc/Dec and Fig. 5c shows the corresponding acceleration. Also, the velocity curve from a nonlinear Acc/Dec is shown in Fig. 5d and the corresponding acceleration in Fig. 5e. Note that no matter what kind of Acc/Dec is used, the final positions are all convergent to final position (this may be verified by calculating the areas of input and output velocity profiles) and the velocity profiles are smooth without oscillation.

Eq. (2) obviously indicates that the structure of the Acc/Dec processor is the same as the finiteimpulse-response (FIR) digital filter. We now define 


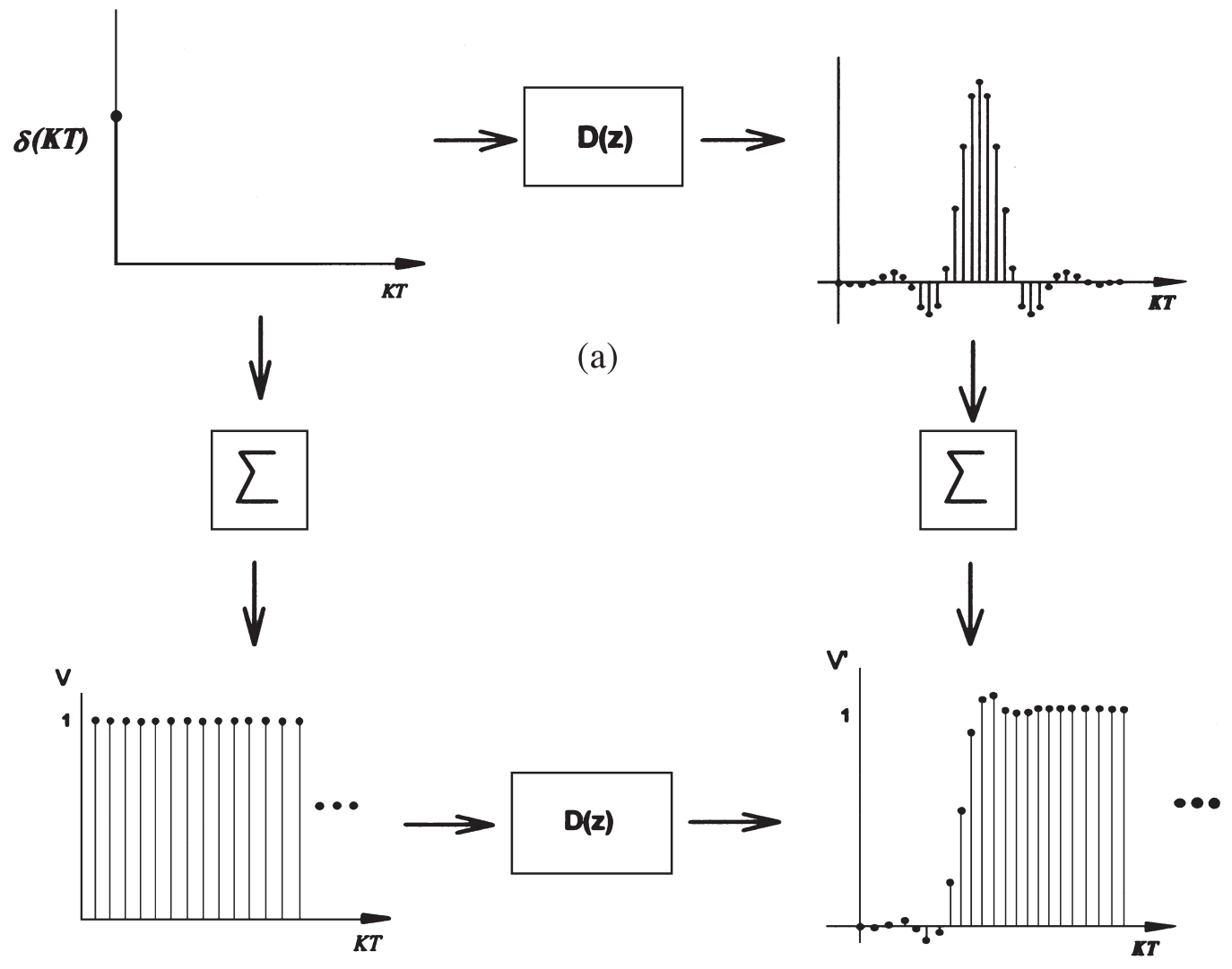

(b)

Fig. 4. Oscillatory impulse response and velocity profile for the Acc/Dec. (a) Impulse and impulse response for the Acc/Dec. (b) Input and output of velocity profile for the Acc/Dec.

$$
D(z) \equiv \frac{V^{\prime}(z)}{V(z)}=\frac{1}{b_{s}} \sum_{i=0}^{N} b_{i} \cdot z^{-i}
$$

where $\mathrm{N}$ is the order of the filter, and Acc/Dec time constant $\tau$ is equal to $(\mathrm{N}+1) \mathrm{T}$. In real implementation, we must spend memory to store the weighted coefficients and the recent velocity commands $(V[k], V[k-1], V[k-2], \ldots . . V[k-n-1])$. Since the coefficients of FIR filter (i.e., the weighted coefficients of Acc/Dec) are usually symmetric, the structures of FIR filter can further be simplified. The simplified structures with odd order and even order presented by signal flow graphs are shown in Fig. $6 \mathrm{~b}$ and $6 \mathrm{c}$, respectively, along with the original structure in Fig. 6a. These simplified structures not only save memory but also reduce computation load.

Consequently, we can design the Acc/Dec processor by the well developed discrete time FIR filter design techniques with some necessary conditions. As mentioned in Section 2, the Acc/Dec after interpolation cause contouring error;. We will presently analyze this induced error in the next section. 


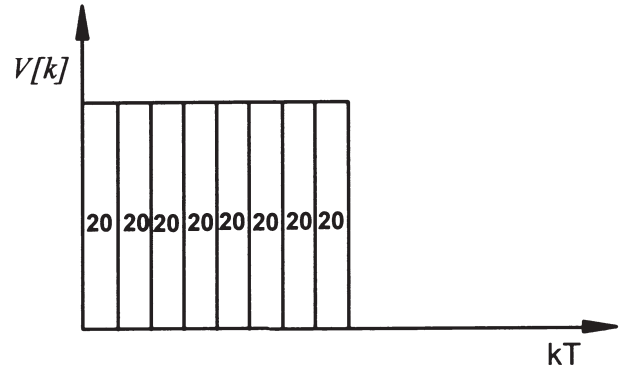

(a)

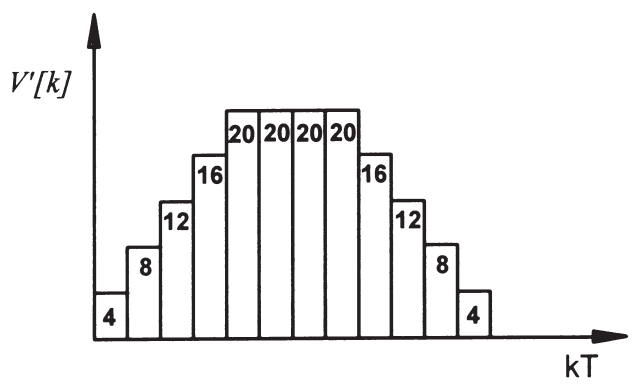

(b)

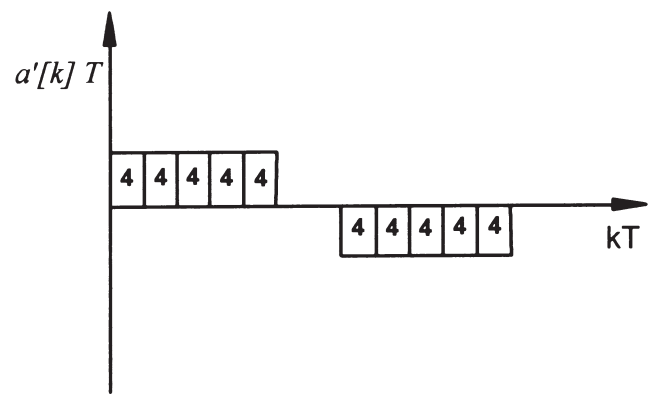

(c)

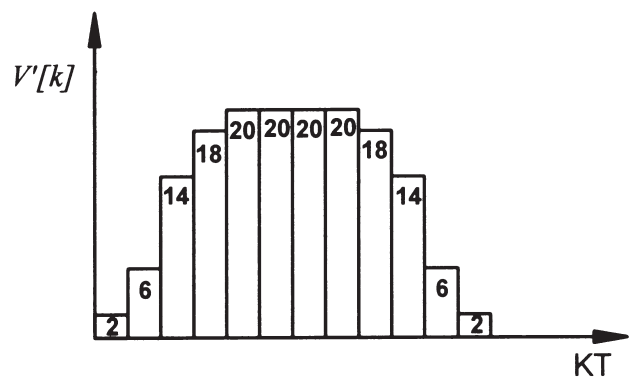

(d)

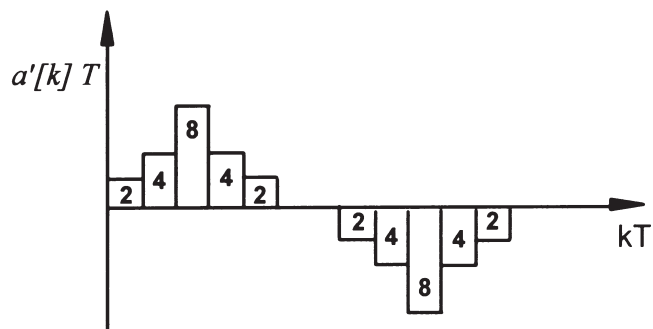

(e)

Fig. 5. Illustrative examples of the proposed Acc/Dec Scheme. (a) Velocity command. (b) Velocity output for $\mathrm{n}=5$, $k_{i}=1(i=1 \sim 5)$. (c) Acceleration of (b). (d) Velocity output for $\mathrm{n}=5, k_{i}=(0.5,1,2,1,0.5)$. (e) Acceleration of (d).

\section{Circularity contouring error analysis}

Let us consider the circular interpolation. As illustrated in Fig. 7, the Acc/Dec after interpolation will cause an error of decreased radius. This phenomenon will be investigated in detail in the following. Feeding the $\mathrm{x}$ axis reference velocity command, $V_{x}[k]=R \omega \sin (\omega k T)$, to the Acc/Dec processor as input series, we obtain the resulting output velocity as 


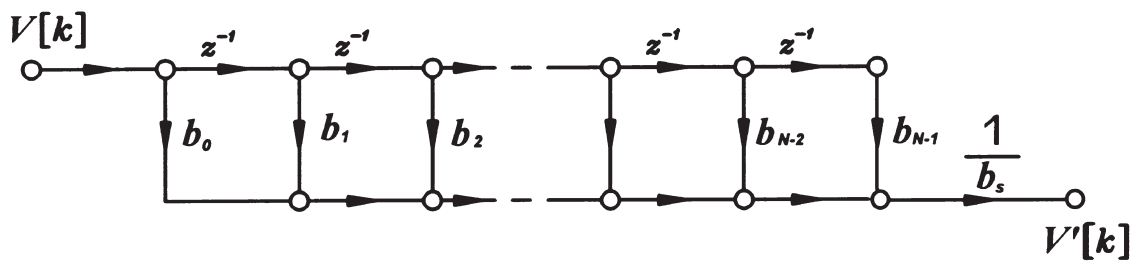

(a)

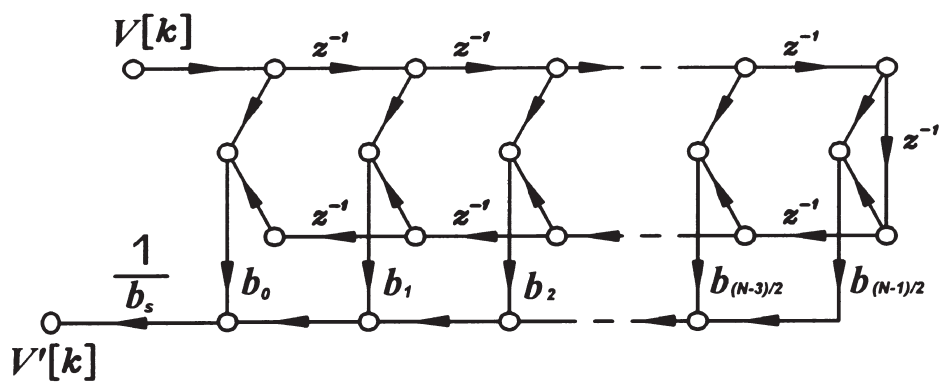

(b)

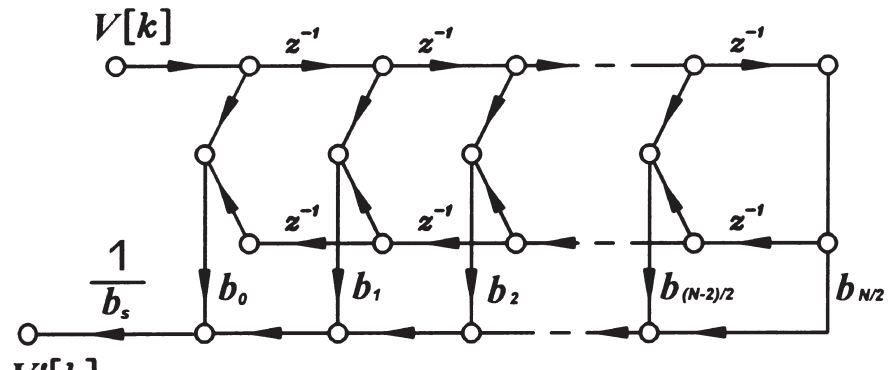

(c)

Fig. 6. Signal flow graphs of digital FIR filters. (a) Original signal flow graphs. (b) Simplified FIR structure of odd order. (c) Simplified FIR structure of even order.

$$
V_{x}^{\prime}[k]=A R \omega \cdot \sin (\omega k T+\phi),
$$

where $A$ is the amplitude frequency response of a FIR filter, i.e., $A=\left|D\left(e^{j \omega}\right)\right|$, and $\phi$ is the phase of the frequency response of a FIR filter. Both are functions of $\omega$. Next, consider the desired position command to the Acc/Dec for the $\mathrm{x}$ axis, which is 


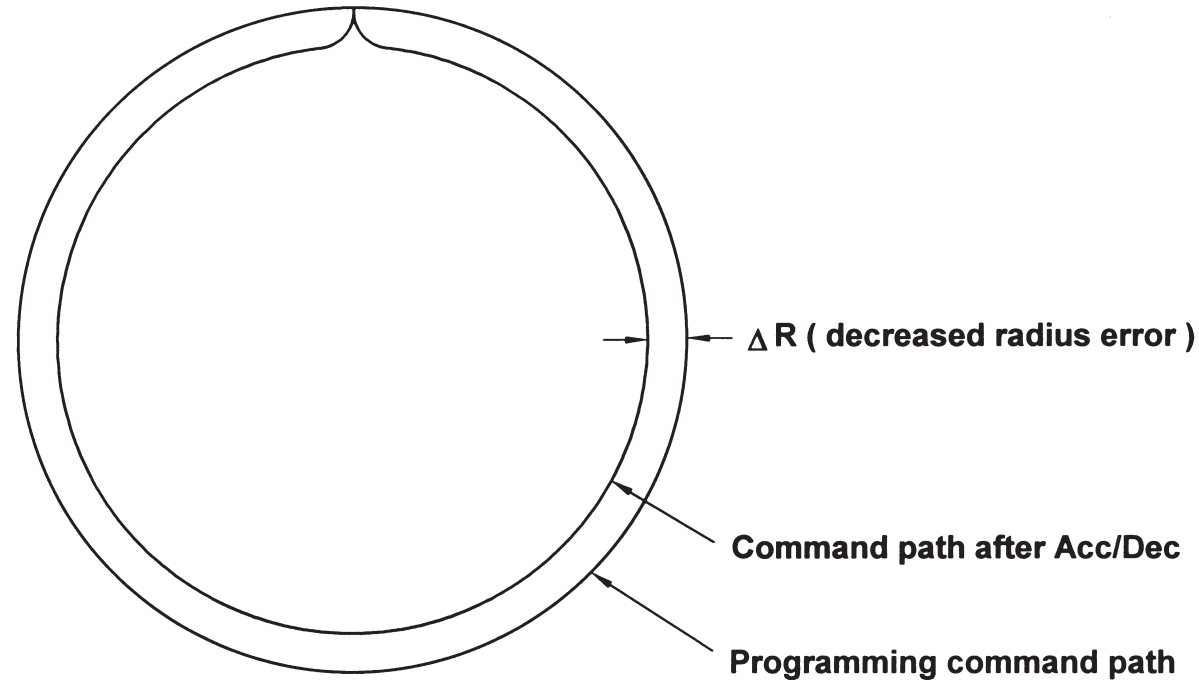

Fig. 7. Error of decreased radius with Acc/Dec after interpolation.

$$
X_{x}[k]=\sum_{j=1}^{k} V_{x}[j] \cdot T
$$

The resulting output position from the Acc/Dec for the $\mathrm{x}$ axis is then

$$
X_{x}^{\prime}[k]=\sum_{j=1}^{k} V_{x}^{\prime}[j] \cdot T,
$$

From the above two equations, it is obvious that the input-output position dynamic is equal to the input-output velocity dynamic, that is,

$$
\frac{X_{x}^{\prime}(z)}{X_{x}(z)}=\frac{V_{x}^{\prime}(z)}{V_{x}(z)}=D(z)
$$

The above result is also applied to the y-axis position dynamic. Since $D\left(e^{j \omega}\right)$ is a low pass FIR filter whose magnitude of $D\left(e^{j \omega}\right)$ is always less than one, the resultant radius in a circular motion will be less than the desired radius and yields a decreased radius error, $\Delta \mathrm{R}$. Note that as far as the contouring error is concerned, the phase lag has no effect, since commands of each axis are fed to the same Acc/Dec processor.

As stated earlier, the coefficients of digital FIR filter are always symmetric; thus, we can deduce a simplified form for the $|\mathrm{D}(\mathrm{z})|$. For the FIR filters of odd order, we have

$$
|D(z)|=\frac{1}{b_{s}}\left|\sum_{i=0}^{N} b_{i} \cdot z^{-i}\right|
$$




$$
\begin{aligned}
& =\frac{1}{b_{s}}\left|z^{-N / 2}\right| \cdot\left|b_{0} z^{N / 2}+\ldots+b_{(N-1) / 2} z^{\frac{1}{2}}+b_{(N+1) / 2} z^{-\frac{1}{2}}+\ldots+b_{N} z^{-N / 2}\right| \\
& =\frac{2}{b_{s}} \sum_{i=0}^{(N-1) / 2} b_{i} \cdot \cos \left(\frac{N}{2}-i\right) \omega T
\end{aligned}
$$

For the FIR filters of even order, we have

$$
\begin{aligned}
|D(z)| & =\frac{1}{b_{s}}\left|z^{-N / 2}\right| \cdot\left|b_{0} z^{N / 2}+\ldots+b_{N / 2}+\ldots+b_{N} z^{-N / 2}\right| \\
& =\frac{1}{b_{s}}\left[b_{N / 2}+2 \cdot \sum_{i=0}^{N / 2-1} b_{i} \cdot \cos \left(\frac{N}{2}-i\right) \omega T\right]
\end{aligned}
$$

Once the coefficients of Acc/Dec curve, $b_{i}$ s, are all determined, Eqs (13) and (14) can be used to calculate the decreased radius error at any working frequency.

Here, we consider the special case of linear Acc/Dec curve, which has the weighted coefficient $b_{i}=1$ and $b_{s}=\mathrm{n}$, or

$$
\begin{aligned}
D(z) & =\frac{1}{n} \sum_{i=0}^{n-1} z^{-i} \\
& =\frac{1}{n}\left(\frac{1-z^{-n}}{1-z^{-1}}\right)
\end{aligned}
$$

Thus, the amplitude frequency response of the FIR filter, A, is

$$
\begin{aligned}
A & =\left|D\left(e^{j \omega}\right)\right| \\
& =\frac{1}{n} \frac{\sin (n \omega T / 2)}{\sin (\omega T / 2)}
\end{aligned}
$$

The working frequency, $\omega$, is usually very small in practical applications, as will be discussed in Section 6. Under this circumstance, Eq. (16) can reasonably be simplified as

$$
A=\frac{1}{n} \frac{\sin (n \omega T / 2)}{\sin (\omega T / 2)} \cong \frac{2}{\tau \omega} \sin (\tau \omega / 2)
$$

If the radius of circular interpolation is $\mathrm{R}$, the radius after Acc/Dec would be

$$
R^{\prime}=\frac{2 R}{\tau \omega} \sin (\tau \omega / 2)
$$




$$
=R-\frac{R(\tau \omega)^{2}}{24}+\frac{R(\tau \omega)^{4}}{1920}-\ldots
$$

By ignoring the high order terms, the radius error becomes

$$
\begin{aligned}
& \Delta R=R-R^{\prime} \\
& \cong \frac{R}{24}(\tau \omega)^{2} \\
& \cong \frac{1}{24 R}\left(\tau V_{\tan }\right)^{2}
\end{aligned}
$$

where $V_{\tan }$ is the tangent velocity. The radius error is dependent upon working frequency, $\omega$. In order to evaluate the usability of approximated radius error formula in Eq. (19), we calculated the radius errors for different working frequencies. Assume the Acc/Dec time constant $\tau=$ $0.041 \mathrm{sec}$, the radius of working space is $15 \mathrm{~cm}$, and the feedrate within $30 \mathrm{~m} / \mathrm{min}$, which is qualified as high speed motion in CNC machine tools. The approximated formula gives very good results, as shown in Table 1.

Up to now, we can draw a conclusion that a low pass FIR filter D(z) with maximum cutoff frequency while within the system limitation is an optimum Acc/Dec velocity profile generator yielding a minimum radius error. Although the contouring error for other complex trajectories cannot be obtained analytically, the above conclusion can also be applied.

\section{Mapping from conventional Acc/Dec to digital FIR filter}

Conventionally, we always describe a Acc/Dec curve by a polynomial. The fatal drawback with the conventional Acc/Dec is that we cannot analyze the contouring error. It is instructive

Table 1

Exact and approximated radius error for circular interpolation

\begin{tabular}{lll}
\hline Feedrate $(\mathrm{mm} / \mathrm{min})$ & Exact error $(\mu \mathrm{m})$ & Approximated error $(\mu \mathrm{m})$ \\
\hline 30000 & 1167.08859501757 & 1167.36111111111 \\
25000 & 810.53601207970 & 810.66743827161 \\
20000 & 518.77332682954 & 518.82716049383 \\
15000 & 291.82324410160 & 291.84027777778 \\
10000 & 129.70342539464 & 129.70679012346 \\
8000 & 83.01096748003 & 83.01234567901 \\
6000 & 4.669400837220 & 4.669444444444 \\
4000 & 2.075300028175 & 2.075308641975 \\
2000 & 0.518826622114 & 0.518827160493 \\
1000 & 0.129706756468 & 0.129706790123 \\
600 & 0.046694440081 & 0.046694444444 \\
400 & 0.020753085550 & 0.020753086419 \\
200 & 0.005188271545 & 0.005188271604 \\
\hline
\end{tabular}


that we map the conventional Acc/Dec curves to the digital FIR filter so that we can access their performance. Now, we present the mapping from two popular Acc/Dec S-curves to their corresponding digital FIR filters.

S-Curve $1 s(t)=3 t^{2}-2 t^{3} \quad 0 \leq t \leq 1$

The Acc/Dec velocity can be discretized as

$$
\left\{\begin{array}{lrl}
V^{\prime}[k]=3 & (k / n)^{2}-2(k / n)^{3} & 1 \leq k \leq n \\
V^{\prime}[k]=1 & k>n
\end{array} .\right.
$$

$\underline{\text { S-Curve } 2} s(t)=10 t^{3}-15 t^{4}+6 t^{5} \quad 0 \leq t \leq 1$

Similar to the S-curve 1 , the discretized Acc/Dec velocity is

$$
\left\{\begin{array}{lc}
V^{\prime}[k]=10(k / n)^{3}-15(k / n)^{4}+6(k / n)^{5} & 1 \leq k \leq n \\
V^{\prime}[k]=1 & k>n
\end{array}\right.
$$

where $\mathrm{n}$ is the number of coefficients. From the normalized Acc/Dec velocity profiles, the normalized acceleration for the S-curve 1 can be deduced as

$$
\left\{\begin{array}{lrl}
a^{\prime}[k]=6(k / n)-6(k / n)^{2} & 1 \leq k \leq n \\
a^{\prime}[k]=0 & k>n
\end{array} .\right.
$$

and the normalized acceleration for the S-curve 2

$$
\left\{\begin{array}{cc}
a^{\prime}[k]=30(k / n)^{2}-60(k / n)^{3}+30(k / n)^{4} & 1 \leq k \leq n \\
a^{\prime}[k]=0 & k>n
\end{array} .\right.
$$

For final position of convergence, by Eq. (7), the corresponding coefficients of the FIR filter are

$$
b_{k-1}=a^{\prime}[k] \cdot b_{s} \cdot T \quad 1 \leq k \leq n .
$$

The number of coefficients is assumed here to be equal to 41. Thus, the coefficients of an equivalent FIR filter can be obtained by Eq. (23). Figure 8 plots the relative curves for the polynomial S-curves and the equivalent FIR filters. Figures $8 \mathrm{a}$ and $8 \mathrm{~b}$ present the normalized velocity and acceleration profiles, respectively. Those figures indicate that the velocity and acceleration of the S-curves and those of the corresponding equivalent FIR filters are almost identical. Besides, Fig. 8c plots the amplitude frequency responses of equivalent FIR filters. The contouring error for each type can then be evaluated by examining these plots.

\section{Acc/Dec design procedures based on digital FIR filter}

The FIR filter design by windowing is a conventional approach [7], and is adopted here for demonstration. Figure 9 presents a flow chart that outline the design procedures for determining 


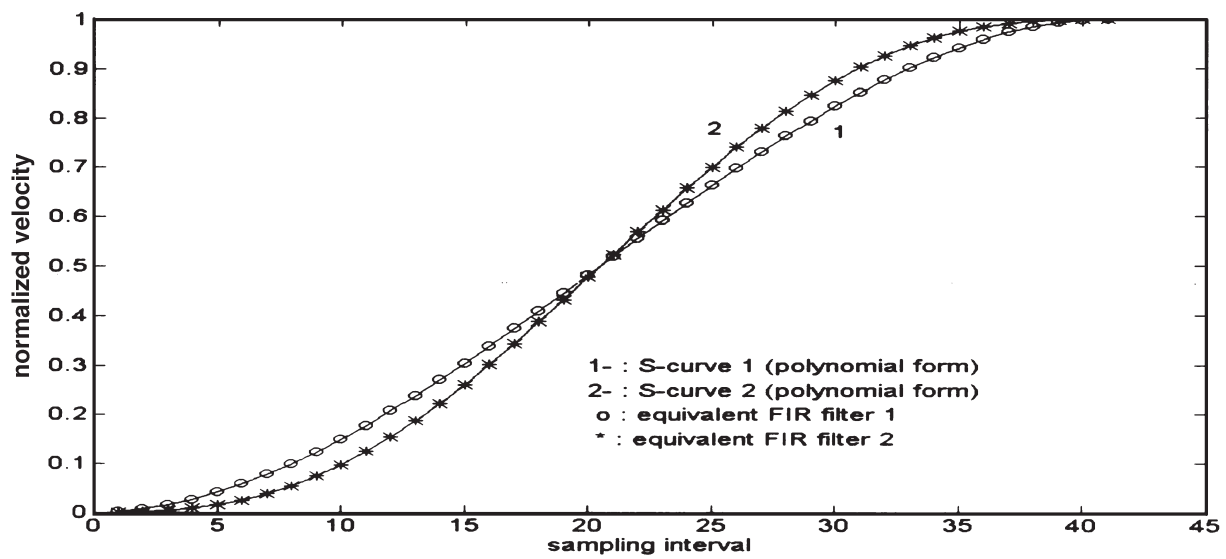

(a)

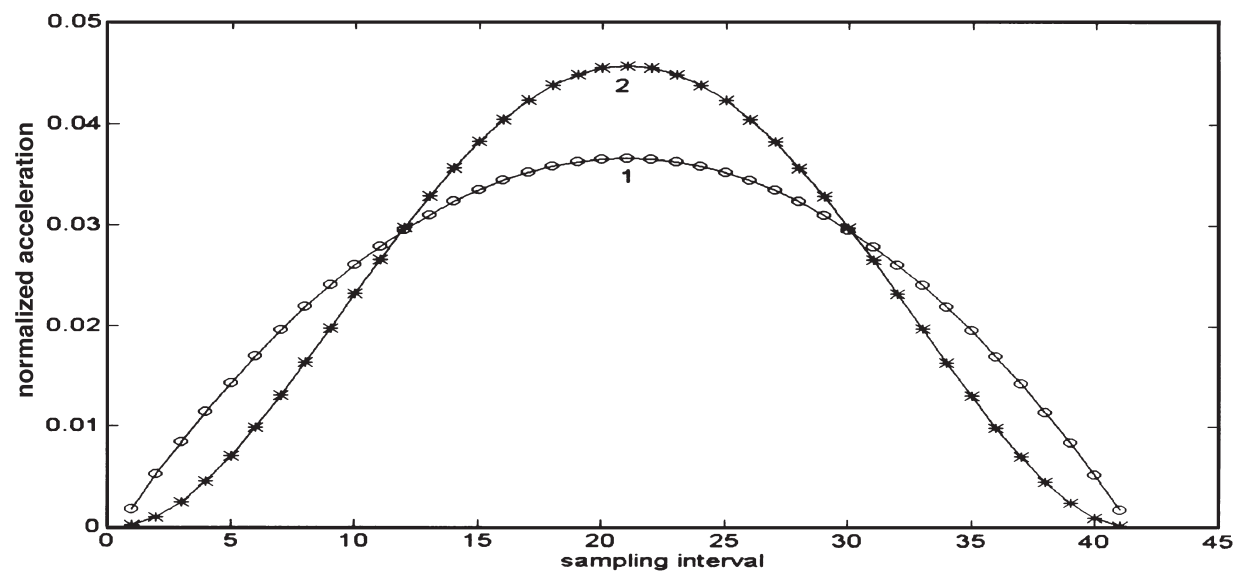

(b)

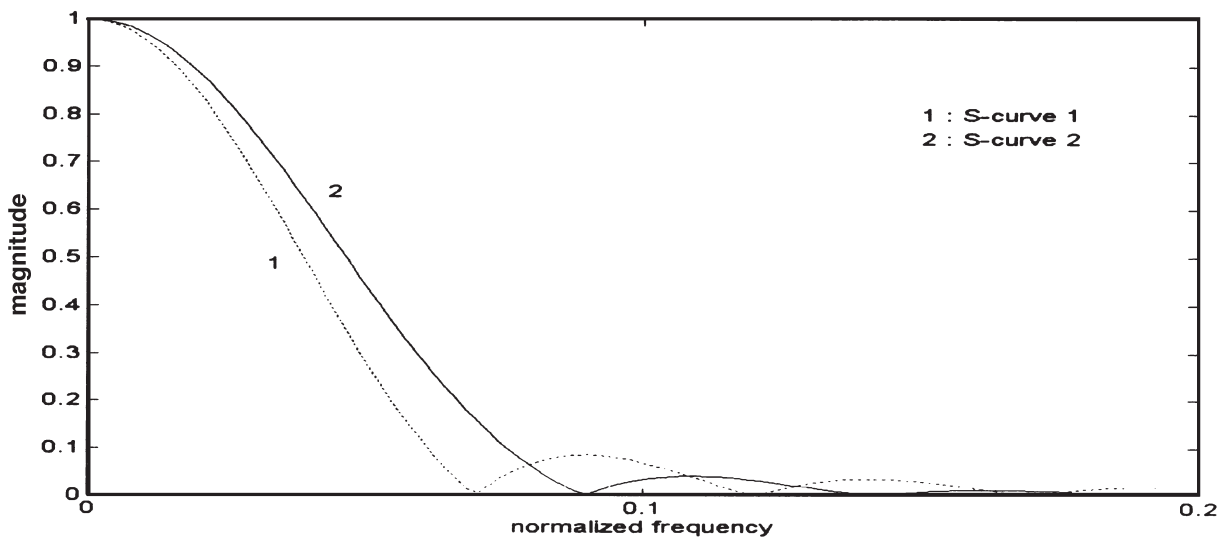

(c)

Fig. 8. Comparisons between S-curves and their equivalent FIR filters. (a) Normalized velocities. (b) Normalized accelerations. (c) The amplitude of the frequency response of the equivalent FIR filters. 


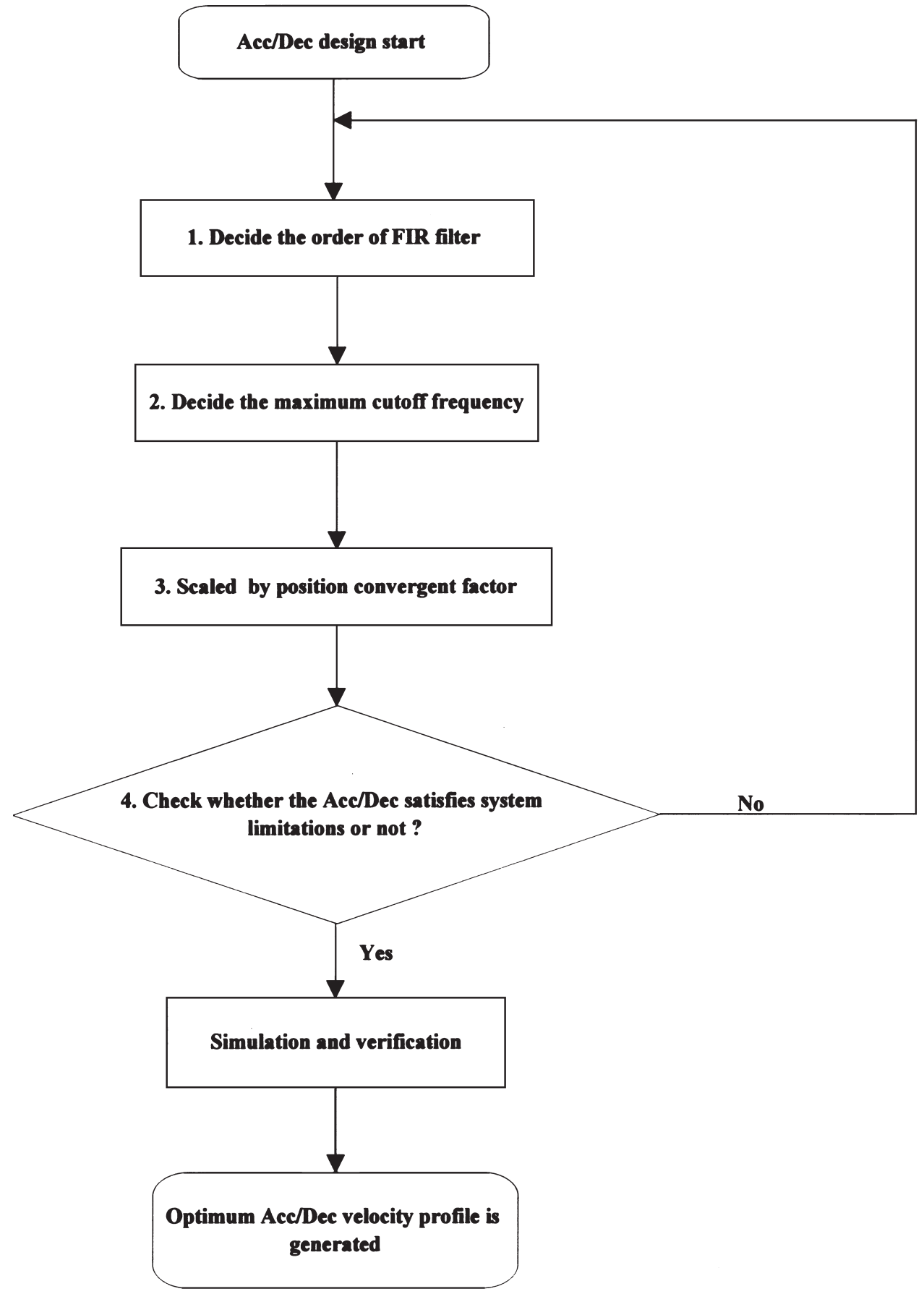

Fig. 9. A flow chart for the Acc/Dec design. 
the optimum Acc/Dec velocity profile. The detailed considerations involved in each step are stated as follows.

\section{Step 1. Determining the order of FIR filter}

The sampling time $\mathrm{T}$ of the closed-loop system should be pre-determined according to such considerations as the system dynamics (i.e., system bandwidth), the complexity of interpolation algorithm and the computer capability. Next, an initial Acc/Dec time constant $\tau_{\text {Acc/Dec }}$ is chosen, which is maintained as small as possible to decrease the BPT and the contouring error. However, too small of a Acc/Dec time constant may cause the driver and actuator to go beyond their limitations. This condition should be checked in step 4. Given the sampling time, the order of the FIR filter can be determined as

$$
N=\frac{\tau_{\text {Acc } / \text { Dec }}}{T}-1
$$

\section{Step 2. Determining the maximum cutoff frequency}

The maximum cutoff frequency $\omega_{n}$ is next determined. Fig. 10a plots the impulse responses of two ideal low-pass filters with time shift $\mathrm{K}_{0} \mathrm{~T}$, and their corresponding amplitude frequency responses can be found in Fig. 10b. Here, the amplitude frequency responses are the same as those of the ideal low-pass filter since the time shift in an impulse response only yields a phase lag. The curve with a sharper main lobe in Fig. 10a corresponds to the filter with a higher cutoff frequency. The cutoff frequency is the inverse of the width of main lobe or the inverse of two times width of side lobes. From step 1 , the order of the filter $\mathrm{N}$ is determined so that the duration of impulse response is fixed, i.e., $(\mathrm{N}+1) \mathrm{T}$. The second condition in Section 2 is recalled, in which the weighted coefficients should be semi-positive. A filter should be selected which has following properties: 1. the impulse response is not oscillatory, and 2. the cutoff frequency is maximum. Consequently, the impulse response should only have a full main lobe with a duration equal to $(\mathrm{N}+1) \mathrm{T}$. If another cutoff frequency, $f_{0}$, higher than $1 /(\mathrm{N}+1) \mathrm{T}$ hertz is selected, the oscillatory pattern appears in its impulse response.

The maximum cutoff frequency is typically represented in a normalized form, i.e., $\bar{\omega}$, with respect to Nyquist frequency $1 / 2 \mathrm{~T}$. Thus, $\bar{\omega}$ can be obtained as

$$
\bar{\omega}=\frac{\frac{1}{(N+1) T}}{\frac{1}{2 T}}=\frac{2}{N+1}
$$

Once the maximum normalized cutoff frequency is determined, the well known window type FIR design method in Signal Processing Toolbox of Matlab [8] can be used to design the Acc/Dec curve. 


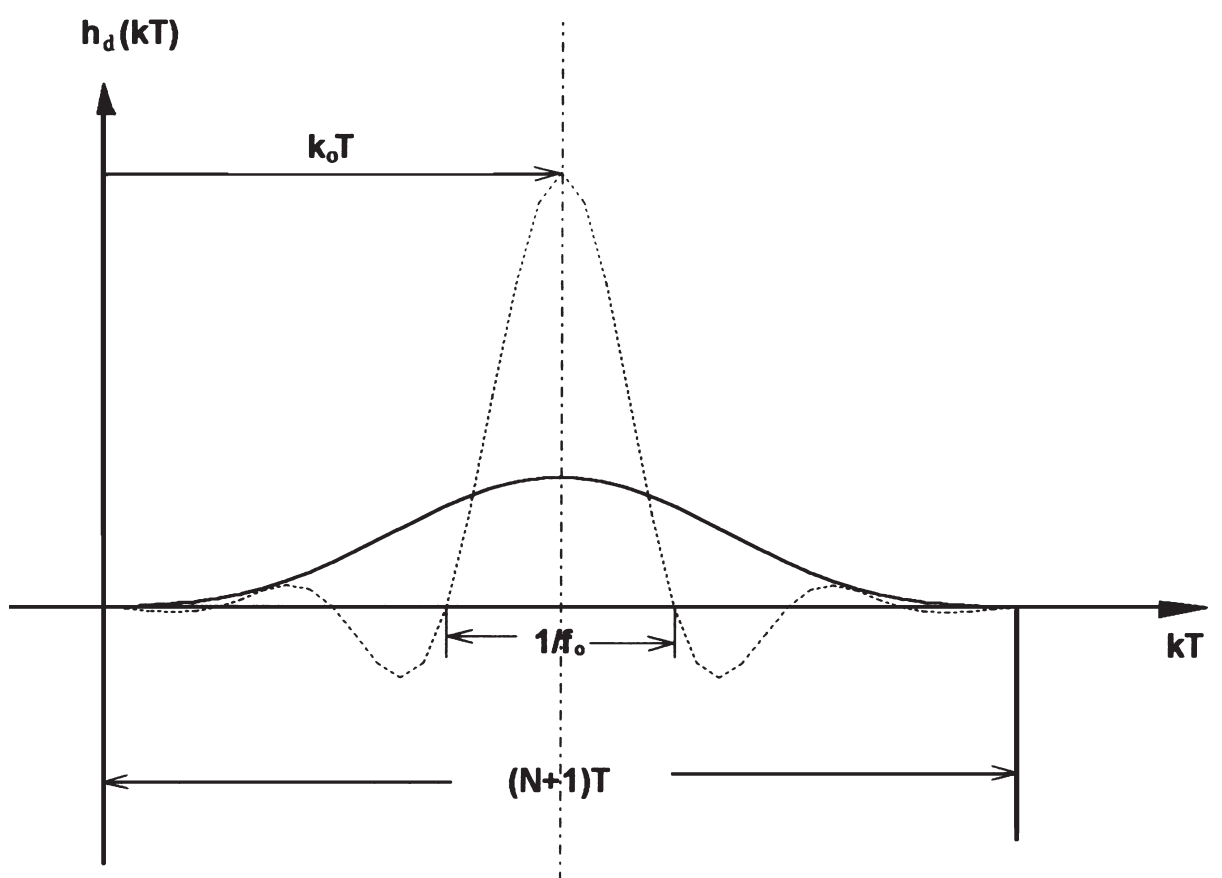

(a)

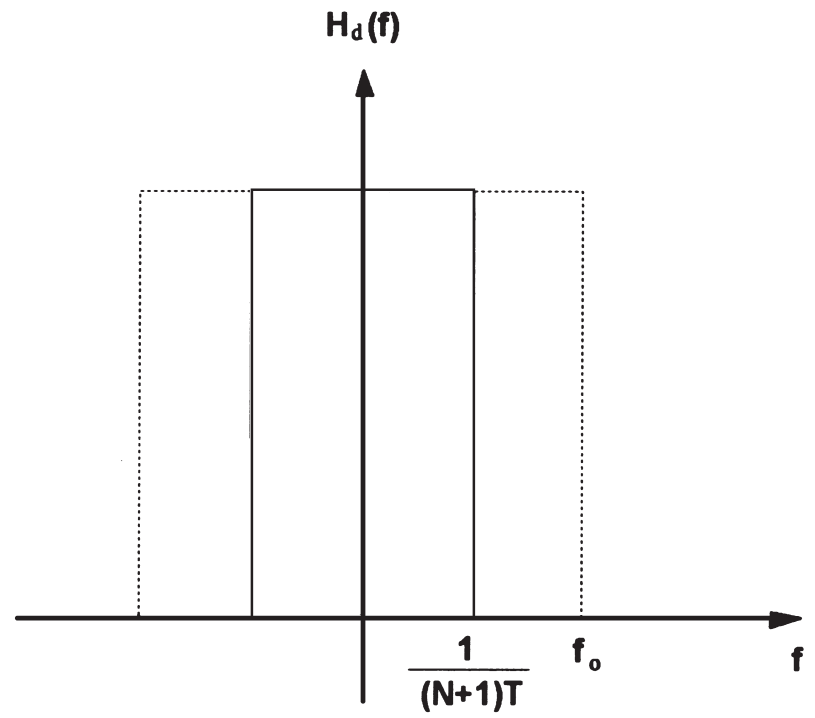

(b)

Fig. 10. Ideal low-pass filters with time shift. (a) Impulse responses. (b) Amplitude frequency responses. 
Step 3. Scaling the FIR filter by the position convergent factor $\mathrm{b}_{\mathrm{s}}$

The final position from the Acc/Dec can be made to converge to the desired position in the motion control by scaling the FIR filter with the position convergent factor $b_{s}$. Eq. (5) in Section 2 provides further details.

\section{Step 4. Checking the system limitations}

Before the final Acc/Dec velocity profile can be determined, all system limitations should be checked. The system limitations include the driver limitation and actuator limitation, where the former includes the current and power limits and the latter includes the force, velocity and power limits. The current limit can be transformed to the equivalent force limit. When using the design Acc/Dec curve, the above system characteristics should be within their limits as follows:

$$
\begin{aligned}
& V^{\prime}[k] \leq V_{\text {Max }} \\
& F[k] \leq F_{\text {Max }} \\
& P[k] \leq P_{\text {Max }} .
\end{aligned}
$$

If these conditions are not satisfied, go back to step 1 and increase Acc/Dec time constant. Then, all procedures are performed again.

The systematic design procedures have already been described as above. Next, the design procedure is demonstrated using an illustrative example in the next section. Furthermore, the result can be evaluated by simulation.

\section{Simulation and results}

In this section, the proposed scheme for the Acc/Dec processor is implemented to carry out simulation studies with an illustrative example. The developed Acc/Dec curve will be compared with conventional Acc/Dec curves including the linear curve, S-curve 1, and S-curve 2. In order to reveal the decreased radius error yielded by Acc/Dec after interpolation with respected to servo series lag. We assume the sampling time $\mathrm{T}=0.004 \mathrm{sec}$ and the Acc/Dec time constant $\tau_{\mathrm{Acc} / \mathrm{Dec}}=$ $0.164 \mathrm{sec}$, then, proceed the design procedures stated in above section:

\section{Step 1. Determining the order of FIR filter}

Substituting the value of sampling time $\mathrm{T}$ and Acc/Dec time constant $\tau$ into Eq. (24) yields the order of the FIR filter.

$$
N=\frac{0.164}{0.004}-1=40
$$


Step 2. Determining the maximum cutoff frequency

As mentioned in the design procedures, the maximum cutoff frequency is determined by Eq. (25)

$$
\omega_{n}=\frac{2}{40+1}=0.049
$$

and then plug it into the Signal Processing Toolbox of Matlab using Blackman window to obtain the optimum weighted coefficients of the Acc/Dec. Fig. 11 displays various curves with different cutoff frequencies. The coefficients with cutoff frequency $\omega_{n}=0.049$ are obviously all positive without any side lobe.

Step 3. Scaling the FIR filter by the position convergent factor $\mathrm{b}_{\mathrm{s}}$

The summation of coefficients of FIR filter, $b_{i}$, designed by the Signal Processing Toolbox of Matlab has already been normalized to one. In this case, $b_{s}$ is equal to one. However, the fact that all $b_{i}$ s are fractional numbers less than one with long digits causes a large truncation error in computation. One approach to overcome this effect is to simply multiply all $b_{i}$ s by a large number then divide by an appropriate $b_{s}$ to guarantee the convergence in the final position.

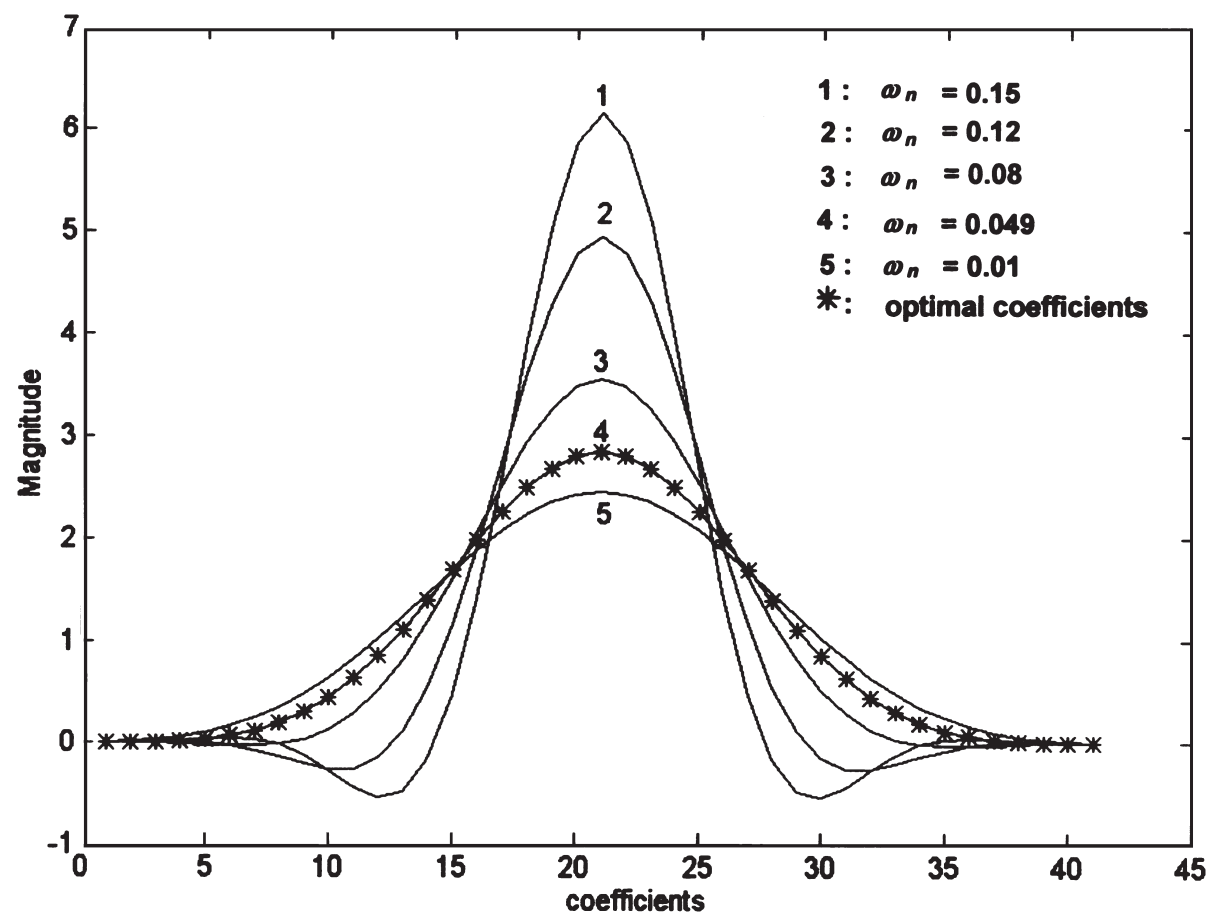

Fig. 11. Coefficients of FIR filters for different cutoff frequency. 


\section{Step 4. Checking the system limitations}

The final design of Acc/Dec curve cannot be accomplished without verifying the system limitations for each axis according to Eq. (26). The maximum overriding velocity is assumed here to be $0.1 \mathrm{~m} / \mathrm{sec}$. Then, by using Eq. (7), the corresponding maximum acceleration would be $7 \mathrm{~m} / \mathrm{sec}^{2}$. Further, assume the equivalent mass and viscous damping of the controlled plant needed for system limitation calculation are $\mathrm{m}=500 \mathrm{~kg}$ and $\mathrm{c}=500 \mathrm{~kg} / \mathrm{sec}$, respectively. Concerning system limitations, the following data are obtained: $V_{\text {Max }}=1 \mathrm{~m} / \mathrm{sec}, F_{\text {Max }}=4000$ Newton, $P_{\operatorname{Max}}$ $=500$ Watts. According to above specifications, the following calculations are proceeded to check the designed Acc/Dec processor.

$$
\begin{aligned}
& V^{\prime}[k]_{\text {Max }}=0.1 \mathrm{~m} / \mathrm{sec}<V_{\text {Max }} \\
& F[k]_{\text {Max }}=500 \times 7+500 \times 0.1=3550 \text { Newton }<F_{\text {Max }} \\
& P[k]_{\text {Max }}=3550 \times 0.1=355 \text { Watts }<P_{\text {Max }}
\end{aligned}
$$

The Acc/Dec design is now complete, which has an order of 40 and a cutoff frequency of 0.049. To compare our design curve with the linear, S-curve 1 and S-curve 2, the following relative information is provided with plots. Fig. 12a shows the coefficients of four Acc/Dec processors, Fig. 12b shows their velocity profiles and Fig. 12c shows their corresponding frequency responses. Note that the comparison is made to examine which of the Acc/Dec profiles is the best in the sense of minimum following error under the circumstances of same Acc/Dec period to achieve a certain target velocity. Our design Acc/Dec curve is obviously the best, having the largest bandwidth. To further evaluate the performance of our design Acc/Dec curve, using the Acc/Dec after interpolator, the reference commands of circular interpolation are fed to linear, $\mathrm{S}$ curve 1, S-curve 2 and our design Acc/Dec processor. Fig. 13 shows the subsequent radius errors for four Acc/Dec processors under a tangent velocity $V_{\tan }=15000 \mathrm{BLU} / \mathrm{sec}$ and radius of motion $\mathrm{R}=4000 \mathrm{BLU}$; the unit of BLU is basic unit length which indicates the encoder resolution in a servo system. The notch portion of the motion profiles corresponds to the system motion during the Acc/Dec period. Our design curve is obviously superior to the others since it has the smallest radius error. Although the results for other complex trajectories are not presented here, adequate performance of our design can be expected since (a) the Acc/Dec is formulated as a dynamic system as stated in Section 2 and (b) our Acc/Dec has the largest bandwidth.

Finally, further comment is made on the Acc/Dec processor. As mentioned in Section 3, the feedrate of $30 \mathrm{~m} / \mathrm{min}$ is qualified as high speed motion in CNC machine tools. The radius of working space is assumed here to be $15 \mathrm{~cm}$; the angular velocity would then be $10 / 3 \mathrm{rad} / \mathrm{sec}$. Correspondingly, it follows that the normalized frequency is

$$
\bar{\omega}=\frac{\frac{\omega}{2 \pi}}{\frac{1}{2 T}}=\frac{T \omega}{\pi}=\frac{10 T}{3 \pi}
$$




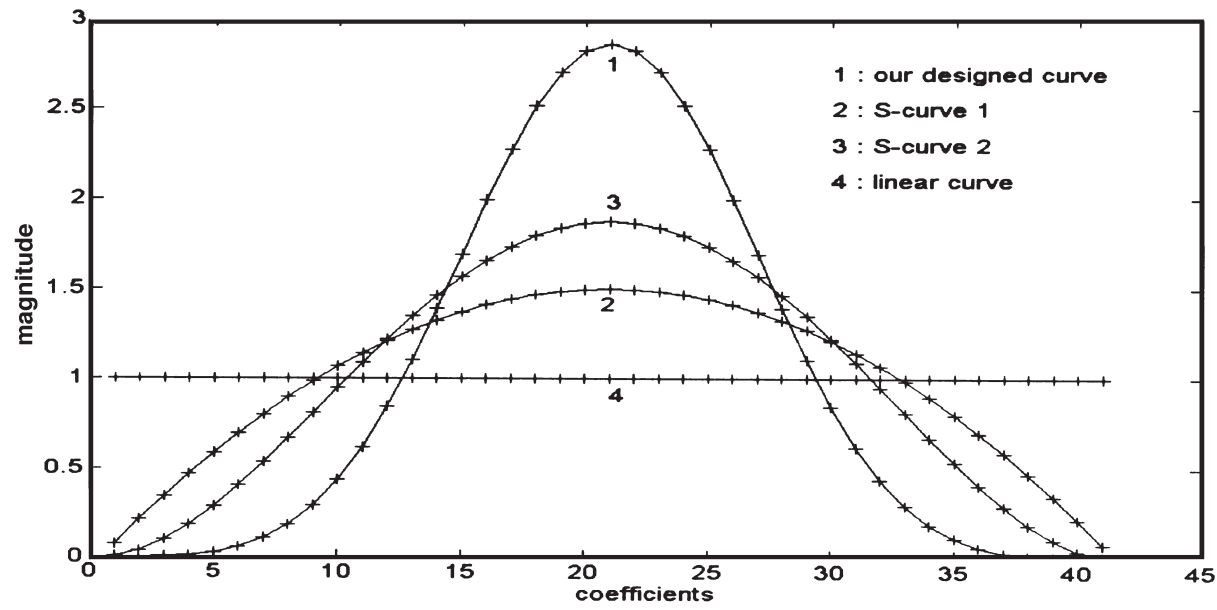

(a)

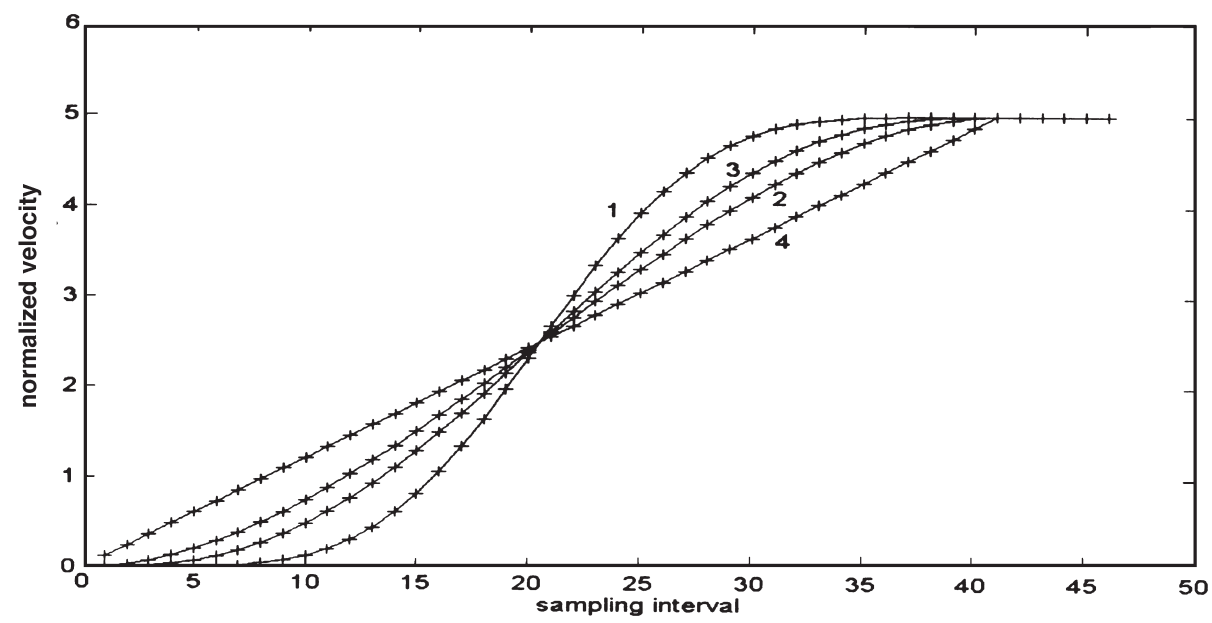

(b)

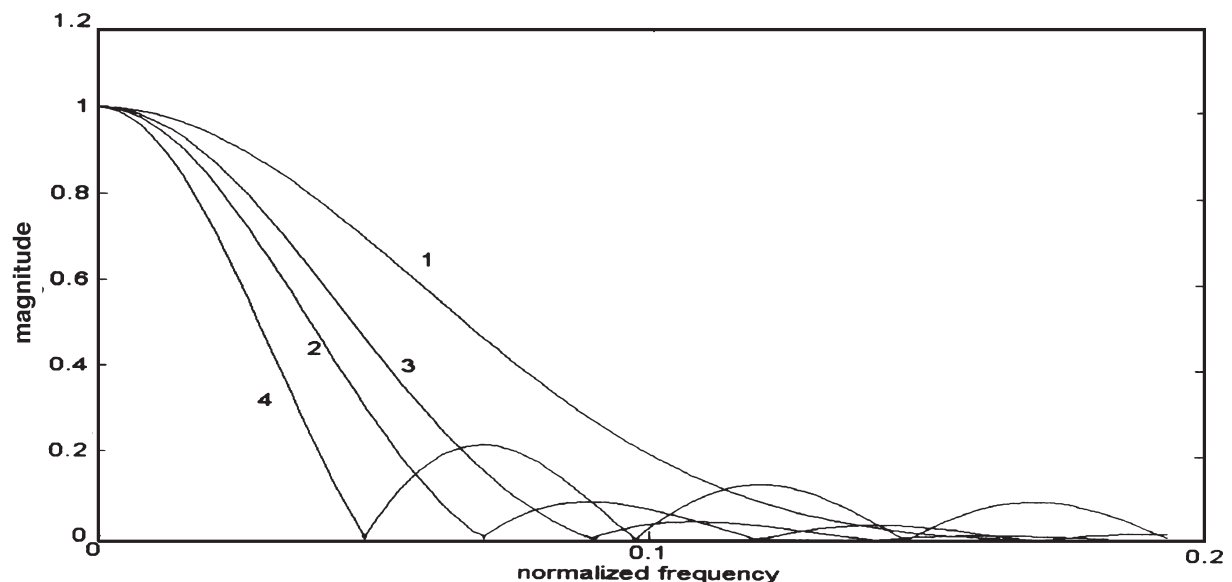

(c)

Fig. 12. Comparisons of different Acc/Dec schemes. (a) Coefficients of the Acc/Dec processor. (b) Acc/Dec velocity profiles. (c) Amplitude frequency responses of FIR filters. 


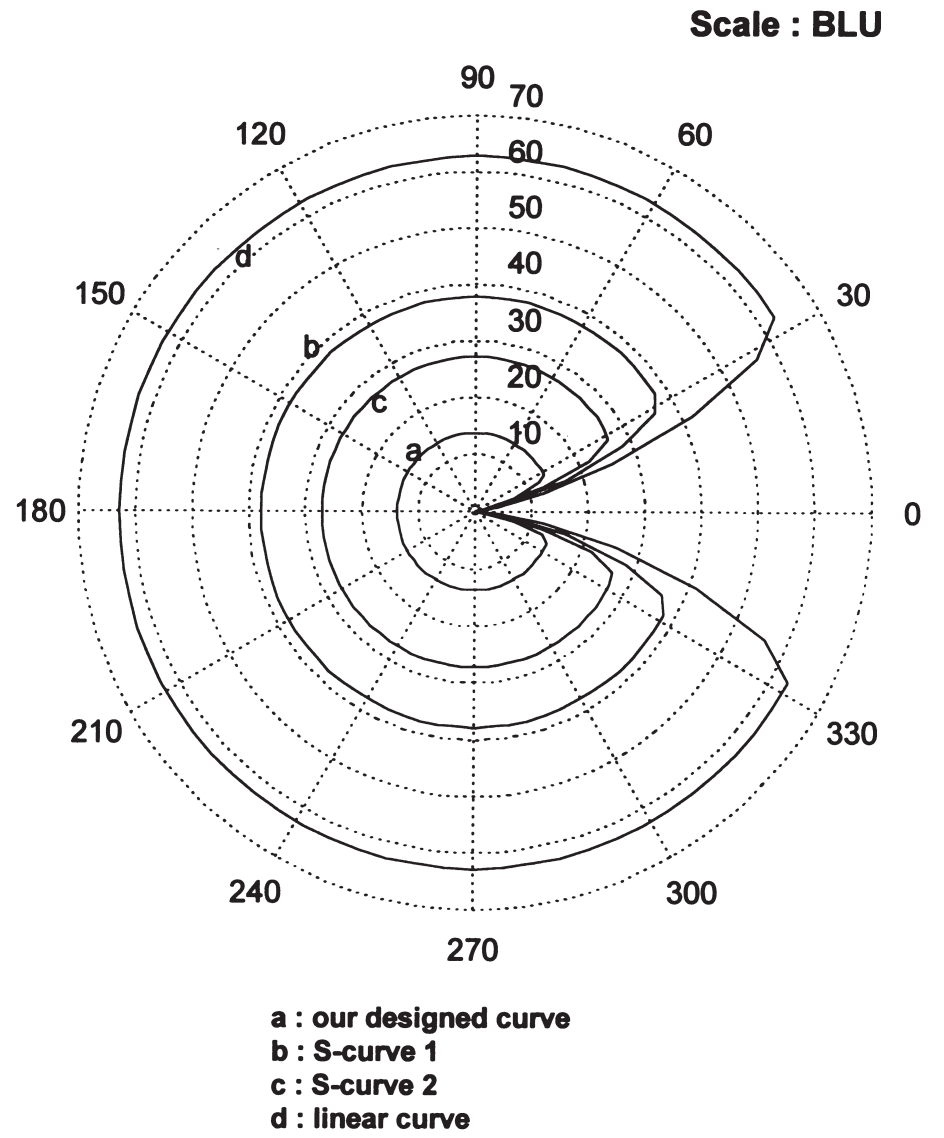

Fig. 13. Radius errors with tangent velocity $15000 \mathrm{BLU} / \mathrm{sec}$ and radius of motion $4000 \mathrm{BLU}$.

If the sampling time of a motion system is $T=0.004 \mathrm{sec}$ as used in the above simulation, the normalized frequency would be $\bar{\omega}=0.0044$. This value is significantly smaller than that of the design cutoff frequency, $\omega_{n}=0.049$. Restated, the nominal operation conditions always fall in the apex region of the frequency response curve. Nevertheless, the induced contouring error in precisely tracking control is quite impressive and should not be overlooked.

\section{Experiments and discussions}

The performance of proposed Acc/Dec processor was tested and confirmed in a retrofitted milling machine. The experiments were conducted under the same motion conditions as those in the simulation study so that the results obtained by both ways can be compared.

\subsection{System configuration}

Figure 14 presents the schematic diagram of the test apparatus and Fig. 15 shows the block diagram of the AC servo drive including a current-loop IP controller and transmission system for 


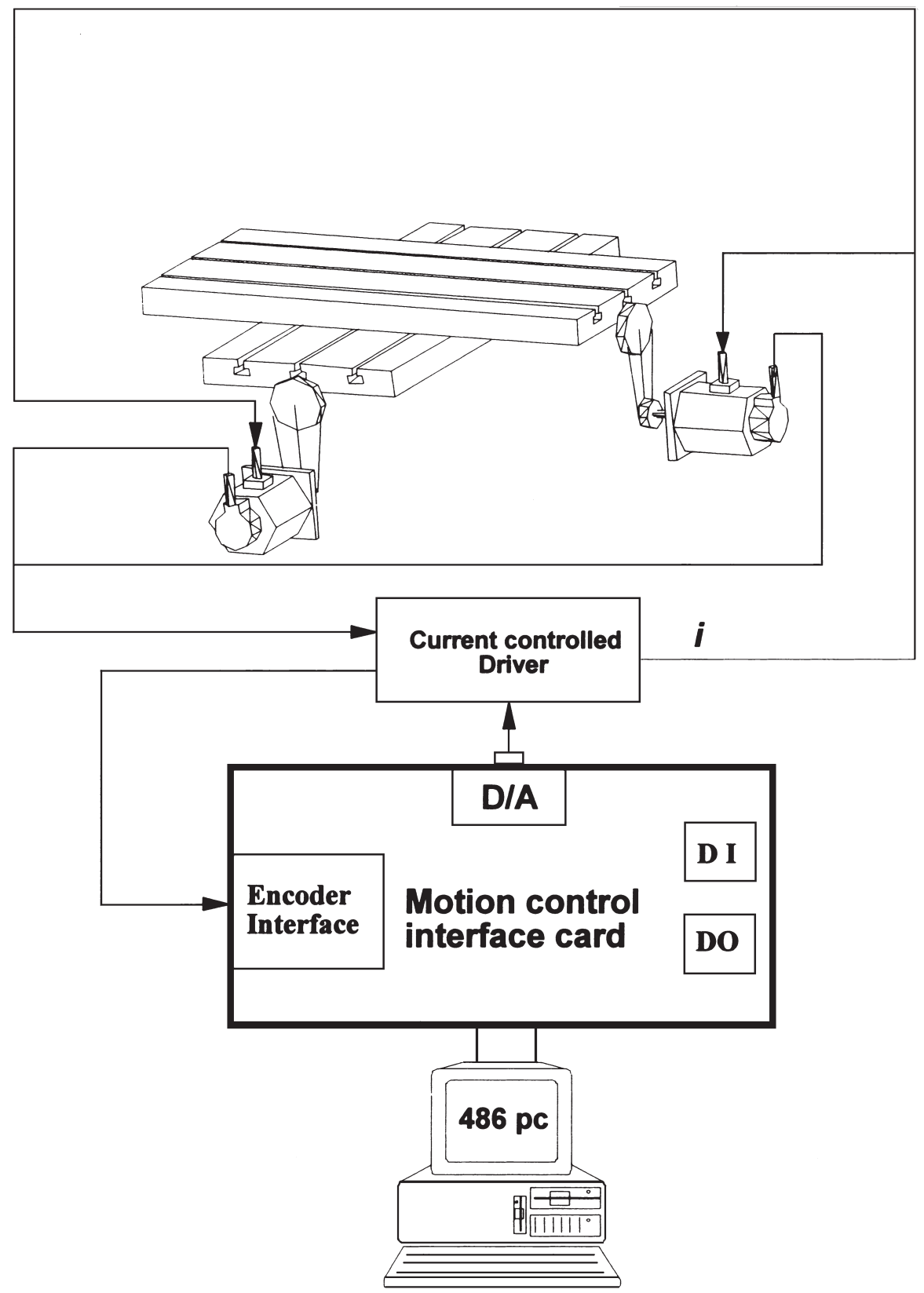

Fig. 14. Schematic diagram of experiment.

single axis. The continuous-time transfer function from the current command $i^{*}$ to the motor angular velocity $w$ is of relative order three.

$$
\frac{w(s)}{i^{*}(s)}=\frac{k i_{i} k_{t}}{L J s^{3}+\left(R J+L B+k i_{p} \cdot J\right) s^{2}+\left(R B+k_{i} k_{e}+k i_{i} \cdot J+k i_{i} \cdot B\right) s+k i_{i} \cdot B}
$$




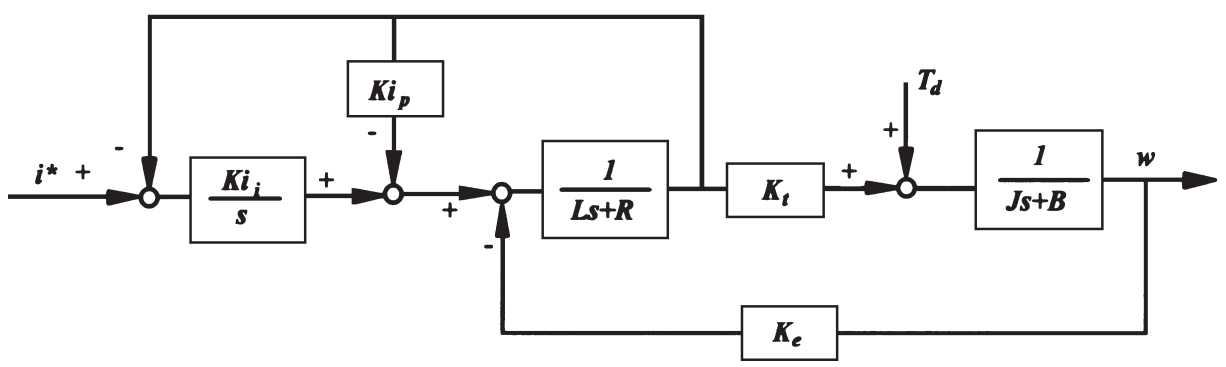

Fig. 15. Block diagram of the AC servo driver system and transmission system.

in which $k_{t}$ is the motor torque constant and $k_{e}$ is the motor back e.m.f. constant; $L$ and $R$ are the inductance and the resistance of the motor stator, respectively; $J$ is the equivalent load inertia (which is a synthetic value of the motor rotor inertia, the pulley inertia and the equivalent table inertia) and $B$ is the equivalent viscous damping (which is a synthetic value of the motor rotor viscous damping, the pulley viscous damping, the belt viscous damping and the equivalent table viscous damping); $k i_{p}$ and $k i_{i}$ are the proportional gain and the integral gain corresponding to the IP current-loop controller.

Although the above transfer function can be derived analytically, the values of the parameters in Eq. (31) are not readily available. Specifically, the equivalent load inertia and the equivalent viscous damping are so complicated that they are hardly evaluated. For ease of controller design, the high gain of current-loop control is always used in practice; consequently, the transfer function can be simplified as a first-order model of

$$
G_{p}(s)=\frac{b}{s+a}
$$

where $a=B / J$ and $b=K_{t} / J[9]$

The frequency response of the above transfer function from the current command (i.e., torque command) input to the angular velocity output was obtained by HP3562 control system analyzer. In the underlying system, the torque command 10 volt corresponds to the motor rated torque $4.802 \mathrm{~N}-\mathrm{m}$ and the angular velocity feedback from encoder is converted into voltage by ONO SOKKI FV-900 F/V converter for further analysis. The estimated models for the $\mathrm{x}$ and $\mathrm{y}$ axes, respectively, are given as follows

$$
\begin{aligned}
& G_{p x}(s)=\frac{33.518}{s+14.45} \\
& G_{p y}(s)=\frac{32.07}{s+13.823}
\end{aligned}
$$

Since the $\mathrm{x}$ axis is mounted on the $\mathrm{y}$ axis, the pole of the transfer function for the $\mathrm{x}$ axis is farther away from the origin than that for the y axis.

The semi-closed loop, i.e., feedback signal from the encoder of servo motor, is employed in 
our servo control system. Figure 16 shows the block diagram of the semi-closed loop servo control system, in which the digital velocity and position controllers are implemented. Here we use the PI velocity controller to handle the velocity loop control. The parameters of the velocity controller, proportional gain $K \nu_{p}$ and integral gain $K \nu_{i}$, are set based on the design specifications that the rise time of velocity response is $33 \mathrm{msec}$ and without overshoot, according to the typical CNC specifications. Specifically, $K \nu_{p}=0.0422, K \nu_{i}=0.0094$ for the $\mathrm{x}$ axis and $K \nu_{p}=0.0442, K \nu_{i}=$ 0.0098 for the $\mathrm{y}$ axis. The position loop controllers for the $\mathrm{x}$ axis and $\mathrm{y}$ axis are only pure gain equaling to $30 \mathrm{sec}^{-1}$

\subsection{Discussions}

In the feed drive dynamic system, the decrease of circle radius may come from two origins, i.e., the Acc/Dec after interpolation and servo delay. Thus, the total decreased radius is the summation of above two effects, or,

$$
\Delta R_{\text {total }}=\Delta R_{\text {servo }}+\Delta R_{\text {Acc } / D e c}
$$

When the position loop gains in the $\mathrm{x}$ axis and $\mathrm{y}$ axis are the same, the decreased radius yielded by servo delay can be approximated as [10]

$$
\Delta R_{\text {servo }} \cong \frac{V_{\tan }^{2}}{2 \cdot R \cdot P G^{2}}
$$

And the term, $\Delta R_{\text {Acc/Dec }}$, is described previously in Section 3.

The experimental results of circular motion with tangent velocity $15000 \mathrm{BLU} / \mathrm{sec}$, radius of motion $4000 \mathrm{BLU} / \mathrm{sec}$ and sampling time $\mathrm{T}=0.004 \mathrm{sec}$ are shown in Fig. 17, where the protrusion error is due to lost motion, occurring from the transmission system including pulley, timing belt and ball screw, and friction between mechanical contact surfaces. The decreased radius from the servo delay, according to Eq. (35), is 31 BLU. If this value is subtracted from the experimental results for each Acc/Dec profile, we will obtain quite similar profiles as those in the simulation study in Fig. 13. It is apparent that our design scheme is superior to the others since it has the smallest radius error.

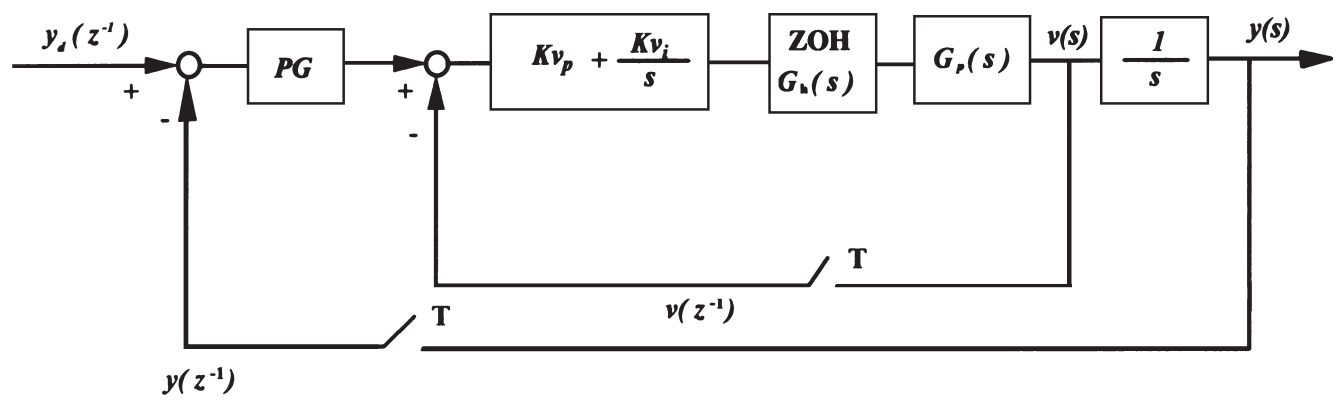

Fig. 16. Semi-closed loop servo control scheme. 
Scale : BLU
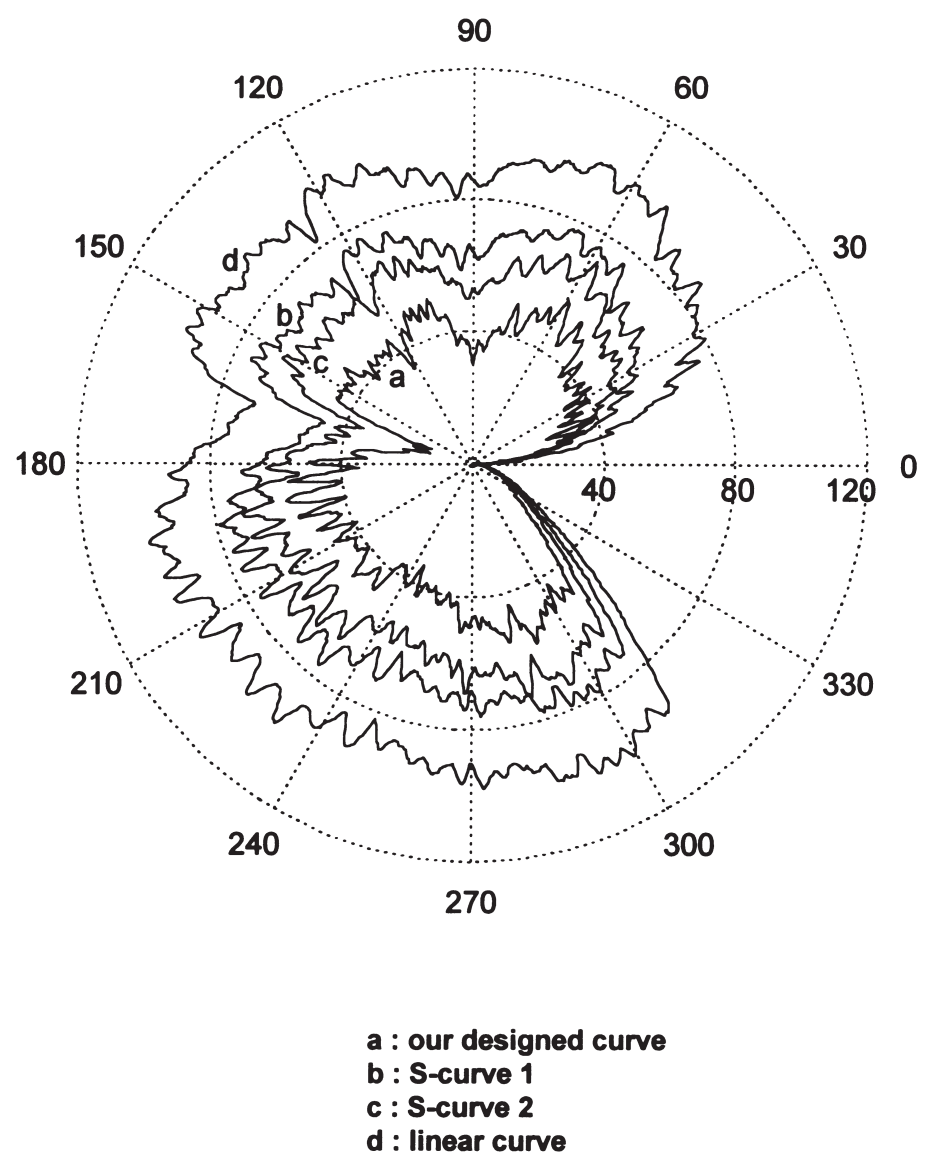

Fig. 17. Experimental results of circular motion with tangent velocity $15000 \mathrm{BLU} / \mathrm{sec}$ and radius of motion $4000 \mathrm{BLU}$.

\section{Conclusion}

This study has presented a relatively simple but novel strategy for the Acc/Dec design, which can be applied to all kinds of motion control applications, e.g., CNC machine tools, and robotic manipulators. A systematic procedure is developed on the basis of the discrete time FIR filter design technique with three necessary conditions, together with the analysis of the contouring error caused by the Acc/Dec scheme. The proposed Acc/Dec algorithm is very flexible in that it creates space for users to use more sophisticated profiles to closely match the system limitations for high performance motion control.

\section{Acknowledgement}

The authors would like to thank the National Science Council of the Republic of China for financial support of this manuscript under Contract No. NSC85-2612-E-009-035 


\section{References}

[1] Y. Koren, Computer Control of Manufacturing System, McGraw-Hill Inc., New York, 1983.

[2] Y. Koren, O. Masory, ASME Journal of Engineering for Industry 103 (1981) 131-136.

[3] O. Masory, Y. Koren, ASME Journal of Engineering for Industry 104 (1982) 400-405.

[4] K.S. Fu, R.C. Gonzalez, and C.S.G. Lee, Robotics: Control, Sensing, Vision, and Intelligence, McGraw-Hill Inc., New York, 1987.

[5] PMAC User's Manual, Delta Tau Data Systems Inc., CA., 1992.

[6] J.S. Park, Mechatronics 6 (1996) 649-663.

[7] V.O. Alan, and W.S. Ronald, Discrete-Time Signal Processing, Prentice Hall Inc, New Jersey, 1989.

[8] N.L. John, and S. Loren, Signal Processing Toolbox User's Guide, Math Works Inc., MA, 1992.

[9] D.Y. Ohm, Intelligent Motion, 26-36, Proceeding, CA, 1990.

[10] C.S. Chen, Error Compensation Techniques of DBB Measurement for Motion Error of NC Tools, Master Degree Thesis, National Chiao Tung University, Taiwan, R.O.C., 1993. 